\title{
Guerres, conflits, transnational et territoire (Partie
} 1)

Didier Bigo

\section{OpenEdition}

12 Journals

Édition électronique

URL : http://journals.openedition.org/conflits/234

DOI : $10.4000 /$ conflits.234

ISSN : $1777-5345$

Éditeur :

CCLS - Centre d'études sur les conflits lilberté et sécurité, L'Harmattan

Édition imprimée

Date de publication : 15 mai 1996

ISSN : 1157-996X

Référence électronique

Didier Bigo, "Guerres, conflits, transnational et territoire (Partie 1) », Cultures \& Conflits [En ligne], 21-22 I printemps-été 1996, mis en ligne le 15 mars 2006, consulté le 30 mars 2021. URL : http:// journals.openedition.org/conflits/234; DOI : https://doi.org/10.4000/conflits.234

Ce document a été généré automatiquement le 30 mars 2021.

Creative Commons License 


\title{
Guerres, conflits, transnational et territoire (Partie 1)
}

\author{
Didier Bigo
}

Certaines formes contemporaines de la conflictualité ont modifié en partie l'économie de notre relation à la guerre sur le plan international ainsi que nos croyances sur les capacités des États à détenir le monopole de la violence légitime sur un territoire donné. Ces formes, ces modalités d'action datent au moins des années 1960 mais elles n'ont été considérées comme réellement menaçantes par les agences de la sécurité (services secrets, armées, polices) que depuis la fin de la bipolarité. Ainsi, entre le monde de la conflictualité et le monde de la sécurité, se pose le problème central de la construction sociale de la menace, c'est à dire la manière dont les agences de sécurité perçoivent à un moment donné les évolutions de la conflictualité et hiérarchisent d'une certaine manière ce qui est à leur yeux important, de ce qui est de l'ordre naturel des choses. Cette approche constructiviste ou intersubjective est souvent peu utilisée par les analystes de la défense et des conflits qui ont tendance à croire majoritairement qu'il existe une représentation objective du monde social (des conflits) qu'il suffit de décrire le plus exactement possible afin de déterminer des chaînes causales permettant de prédire et de prescrire des comportements. Nous faisons ici l'hypothèse que les " discours sur le nouvel ordre ou le désordre international " ne reflètent pas forcément les évolutions de la conflictualité. Il existe une semi-autonomie du monde de la sécurité à l'égard des évolutions de la conflictualité ${ }^{1}$. Les discours contemporains sur l'ordre international ne sont pas le réactif aux évolutions des conflits qui, objectivement, seraient plus nombreux et instaureraient le désordre, ils ont leurs propres normes de construction et déterminent nos visions de ce qu'est, à un moment donné, la conflictualité. Par exemple, les conflits périphériques, les conflits de basse intensité qui étaient négligeables deviennent le signe du désordre international. Dès lors, il existe des décalages (entre les acteurs concernés dits locaux et les acteurs occidentaux dits centraux), des retards de perceptions (croyances dans la primauté de l'État des stratégistes), ou des anticipations qui créent par leurs catégories nouvelles, de nouvelles visions sur l'ordre international joignant ce qui était disjoint (zone grise, turbulences stratégiques) et disjoignant ce qui était insécable auparavant (guerres 
populaires en narco guérilla, terrorisme, crime organisé...). Ces nouvelles visions sur la sécurité, sont alors autant dépendantes des luttes internes entre ces acteurs de la sécurité pour imposer une grille de lecture sur " le sens " du monde que des évolutions contemporaines de la conflictualité. Nous avons analysé le premier temps de cette dynamique dans un article précédent en décrivant comment un certain nombre de " grands récits " (fin de l'Histoire, unipolarité, désordre international, clash civilisationnel) ne se distribuent pas au hasard de l'inventivité de quelques intellectuels, mais selon des logiques de positionnement au sein du monde de la sécurité et selon l'économie des luttes qui s'y déroulent pour la prédominance; prédominance qui tient à la capacité de désigner qui sera le nouvel ennemi, où sont les menaces, et qui fait peur². Ces discours provenant des acteurs de la sécurité créent des effets de sens qui " ordonnent " nos représentations des événements et induisent un certain type de " visibilité " de l'ennemi " désigné " 3 . Ces modifications du regard changent donc le registre du visible et de l'invisible, de l'essentiel et de l'accessoire dans l'ordre de la conflictualité, et distribuent autrement ce qu'est une menace " sérieuse " parmi les phénomènes de violence et de conflits. Ainsi, le regard stratégique se transforme mais ces transformations ne reflètent pas celles de la conflictualité. Nous voudrions ici revenir sur le deuxième temps de la dynamique de la construction sociale de la menace, et montrer comment on peut penser les formes contemporaines de la violence. Certaines formes de conflit n'ont plus grand chose en commun avec les représentations traditionnelles que nous avons de la figure de la guerre comme conflit interétatique et conquête territoriale ${ }^{4}$. La capillarisation de la violence, la transnationalité et la contiguïté virtuelle des territoires, la multiplicité des acteurs et leur relative invisibilité, la transformation des rapports de " voisinage " et le jeu des réseaux modifient les enjeux du conflit où la lutte pour la reconnaissance s'articule de manière différente avec le rapport au territoire et n'est pas pour autant le signe d'un clash civilisationnel ou d'un nouveau désordre international. Conflits contemporains et matrice de la guerre Depuis toujours, penser la conflictualité a été pour les stratèges, penser la guerre ${ }^{5}$. Seule celle-ci, majeure, car au fondement même de la survie de la communauté politique, était digne d'intérêt. La guerre entre États n'était certes pas toute la conflictualité mais la réflexion sur la guerre était la matrice des réflexions sur les autres conflits. En expliquant la guerre, on expliquait de facto ces petites guerres que sont les guérillas, et ces guerres internes que sont les guerres civiles et les révolutions. Au delà du moyen de la violence physique par des collectifs organisés, c'est le principe de la polarisation duelle ami-ennemi qui faisait de ces derniers cas des sousproduits des conditions de la guerre.

2 La matrice guerrière et la dualisation Bien que l'on puisse déterminer deux archétypes : la guerre dans l'ordre externe et l'arbitrage dans l'ordre interne, toute remise en cause du monopole de la violence dans l'ordre interne était perçue comme la polarisation duelle de la société et le principe d'une montée aux extrêmes entre deux camps empêchant les situations de tiers indifférents et de possibilité d'arbitrage par un tiers. La matrice guerrière s'infiltrait donc dans la société. Ainsi, loin d'une symétrie entre deux ordres, la guerre et l'arbitrage, penser la conflictualité et la violence, a toujours été, au moins pour le paradigme réaliste (et aronien), donner la priorité à la guerre, donner la priorité au duel sur le triangulaire. La guerre, même générale et totale, dualise le monde. La Révolution fait de même. Lénine a lu Clausewitz. Guerres interétatiques et (grandes) Révolutions semblent ainsi être les deux pôles explicatifs du continuum conflictuel pour les conflits utilisant la violence physique comme modalité 
d'action au sein d'un répertoire diversifié Nous avons discuté cette notion de continuum conflictuel à l'interface de l'ordre interne et de l'ordre international et remettant partiellement en cause la thèse d'une distinction de nature entre ordre interne et ordre externe dont Raymond Aron fut en son temps le plus brillant défenseur. Didier Bigo, " La conflictualité à travers l'analyse de la banque de données de l'Institut français de Polémologie " in Approches polémologiques, FEDN, Juin 1991. ]]. La matrice de la dualité fonctionne sur l'ensemble du continuum et traverse l'interne et l'externe.

3 La matrice guerrière et le rapport au territoire Seulement utiliser la matrice de la guerre conduit, outre la réduction au duel, à une deuxième réduction tendancielle du champ ou du continuum conflictuel, celle de ne voir que les conflits liés à l'Avoir, à la possession, à l'acquisition territoriale. Le conflit aurait sa source dans le désir d'avoir " sa " terre et dans la volonté d'en agrandir l'étendue en fonction de la démographie ou de la volonté de puissance disent les polémologues ${ }^{6}$. La guerre marquerait l'espace dans la mesure où les collectifs se veulent homogènes et structurent en partie leurs identités de manière polémique afin de chasser le traitre à l'intérieur et de tenir les frontières contre l'ennemi extérieur. Au delà des guerres de religion, des guerres de reconnaissance, les guerres inter-étatiques (et inter-nationales?) seraient donc celles qui ont voulu la contiguïté et l'homogénéité permettant la possession et la jouissance entre " soi " d'une terre. La fonction de contiguïté territoriale importe alors plus que l'étendue du territoire conquis. Les guerres européennes changeront de configuration dès le milieu du 17ème siècle pour répondre à ce nouvel " impératif territorial " 7. Aux empires territorialement disjoints et unis symboliquement par l'appartenance au souverain, succéderont des luttes pour agrandir les frontières. Les guerres étatiques seront des guerres de " frontières ". Les dynamiques de la contrainte et du capital favoriseront d'ailleurs les formes étatiques dont la combinaison sera la plus équilibrée au détriment des empires à faible capital ou des ligues de villes à faible capacité coercitive $^{8}$. Le territoire se déclinera à partir de ce moment comme un construit social et politique " encageant " les populations, permettant de les contrôler, de les surveiller ou au moins de les influencer ${ }^{9} .{ }^{10}{ }^{11}$. Cette forme étatique de la gouvernementalité européenne a alors déterminé comme horizon un programme de " pureté ", d'exclusion des Tiers dont la territorialisation, la " frontaliérisation " est le marquage particulier de l'espace. Plus tard, certains régimes voudront aller jusqu'au bout de cette logique d'une territorialité homogène (l'Allemagne nazie, et plus proche de nous, la Grande Serbie) mais les autres se contenteront d'un enjeu symbolique et refuseront de s'épuiser à exclure les différences. En pratique, les gouvernements se contenteront d'une obéissance banale et toléreront la diversité du vivre-ensemble. Les processus de démocratisation se feront même au nom de cette valeur de la diversité et si cela " désacralisera " quelque peu le territoire, cela renforcera le respect pour l'autre. L'allégeance citoyenne supposera une acceptation des règles de procédure majoritaire élective et un minimum de croyances dont celle de tolérance à l'égard des idées opposées. Seulement comme le soulignent avec force, Bertrand Badie et Marie-Claude Smouts, ces rapports complexes entre guerre, territoire, État, démocratie, citoyenneté changent. Certaines tendances lourdes de la transnationalité érodent cette conception $\mathrm{du}$ vivre ensemble et exacerbent la territorialisation du politique qui semble plus présente que jamais. Pire, le programme de pureté fait sa réapparition et la guerre des cultures est soi-disant notre horizon ultime. En même temps, les conflits contemporains ne sont plus majoritairement des guerres interétatiques ou de grandes 
révolutions ${ }^{12}$. La polarisation duelle (de la guerre ou de la révolution) semble ne plus véritablement rendre compte de multiples formes de conflits. L'espoir mis dans la rationalité des acteurs, la théorie des jeux pour obtenir une étude scientifique des comportements de guerre a produit l'inverse de ce qui était espéré. On a réduit la " guerre caméléon " à la " guerre théorie des jeux " au lieu d'expliquer par cette dernière et ses raffinements théoriques, tous les types de comportements guerriers, ce qui était le projet initial. On a réduit la conflictualité à des luttes pour l'Avoir en déniant au conflit la capacité de structurer des identités qui ne lui préexistent pas forcément. Or, même en admettant que durant un temps, pour des raisons heuristiques, ces réductions, ces simplifications de la réalité, ces modélisations se justifiaient, actuellement, la dissémination de la violence, sa capillarisation ont changé les conditions de son emploi et ont multiplié les cas où la violence n'est plus en relation directe, ni avec un affrontement tendanciellement duel, ni avec des buts de conquête territoriale.

Déjà mise à mal par la dissuasion comme non guerre, ou guerre virtuelle, la matrice guerrière semble, à nos yeux, incapable d'expliquer les raisons des formes fragmentées, aléatoires, disséminées de la conflictualité contemporaine (fragmentation quasi infinitésimale des entrepreneurs de violence sur un territoire donné, dislocation des grands espaces en formes locales, extension en revanche des réseaux de solidarité au delà de l'espace conflictuel direct... Cf. Liban, Somalie...). Les principes d'affrontement de volontés entre deux acteurs bien définis, de connaissance et de reconnaissance mutuelle des enjeux de la guerre, d'économie des forces pour la conquête d'un territoire qui sont l'encadrement méta-théorique de la stratégie militaire ne semblent plus s'appliquer avec la même pertinence. De même la Révolution n'est plus à même d'expliquer toutes les formes infra-conflictuelles internes. Les actions collectives violentes ne sont pas toutes des tentatives révolutionnaires avortées. Même en élargissant comme Charles Tilly le spectre révolutionnaire aux petites révolutions et aux coups d'État on ne peut subsumer la diversité de ces actions. Certaines formes conflictuelles (internes, externes, transnationales) semblent irréductibles à la polarisation duelle de forces pour la conquête du pouvoir sur un territoire donné.

Des formes nouvelles de conflictualité ? Certes, si nous regardons les listes des conflits contemporains à notre disposition, nous voyons bien que nombre de conflits ressortent toujours de la guerre et de la polarisation duelle ${ }^{13}$. La guerre pour une querelle frontalière n'est pas morte comme le montre le récent conflit entre le Pérou et l'Équateur. Et l'on peut affirmer sans trop de risque que les logiques de puissance, de conquêtes territoriales ne sont pas prêtes de s'arrêter. D'ailleurs, qu'une partie fondamentale des guerres de l'ex-Yougoslavie soit explicable par les luttes pour la conquête territoriale est sans contestation, même si résumer à cela l'épisode bosniaque serait sans doute bien rapide. Que les conflits dits internes du Tchad, d'Éthiopie, du Rwanda, du Tadjikistan, d'Afghanistan, de Géorgie, de Tchetchénie, d'Inde, du Sri Lanka, du Timor...aient une composante territoriale déterminante pour ceux que l'on appellent les " séparatistes " ne fait aucun doute. Mais jusqu'où cette vision du conflit explique-t-elle véritablement la Tchetchénie ou le combat Kurde ou les luttes en Algérie et en Colombie ? Peut-on analyser le Liberia, le Pérou, la Somalie à travers cette grille de lecture de la polarisation duelle? Qu'en est-il des formes de violence qui affectent l'espace occidental et qu'on nomme terrorisme international, maintenant qu'il est difficile d'y voir le jeu de stratégies indirectes du camp soviétique ? Le regard se transforme et avec lui " apparaît " une lecture différente de la conflictualité. Non pas 
qu'il s'agisse ici de substituer une explication à une autre. Il s'agit uniquement d'attirer l'attention sur les erreurs possibles d'analyse qui se produisent lorsqu'on lit à travers la matrice de la guerre les diverses luttes pour la reconnaissance, les diverses logiques conflictuelles. La matrice de la guerre et de la polarisation duelle explique donc encore très largement les conflits majeurs que nous connaissons, mais ce qui pose problème à l'analyse ce sont les autres formes de conflits, celles qui ne sont labellisables ni en terme de guerres, ni en terme de stratégies indirectes menées par deux acteurs bien identifiés, ni en terme de tentatives de révolutions.

Ces divers conflits, qu'on les labellise comme turbulences stratégiques, comme conflits de basse intensité, comme zones grises, comme narco-criminalité, comme violences urbaines, comme terrorisme ne relèvent pas des mêmes logiques, et en particulier n'ont pas forcément le même rapport au territoire. S'inscrire sur un espace géographique reste certes un enjeu pour les organisations les plus puissantes, là où la dissymétrie avec les forces au pouvoir est la moins grande mais pour les autres groupes, qu'en est-il ? Ils ne visent pas forcément à homogénéiser un territoire qu'ils sont incapables de conquérir par la force et à le frontaliériser avec une délimitation claire entre un intérieur et un extérieur. Le processus géopolitique de la frontaliérisation homogénéisant la population et expulsant l'ennemi hors du territoire n'est pas, ou pas tout le temps celui qui conduit leurs logiques d'actions. La multiplicité d'acteurs infra-étatiques ou libres de souveraineté, la prééminence de logiques micro sur la conquête du pouvoir, l'invisibilisation tactique de certains acteurs, la dimension sacrificielle de certaines stratégies, l'intersubjectivité des luttes pour la reconnaissance et la délocalisation des combats ainsi que le rôle du transnational, des media, de la vitesse de communication, de la temporalité, des nouveaux rapports de voisinage et d'inimitiés sont autant d'éléments qui peuvent perturber le rapport du conflit à l'espace et au territoire. Et ceci n'est pas neuf, ce sont des caractéristiques qui datent au moins des années 1960 mais si les polémologues les avaient signalées, les stratégistes n'étaient pas prêts à l'admettre avant l'ébranlement de leurs certitudes, dans les années 1990.

7 Des acteurs multiples et hétérogènes La multiplicité d'acteurs, qui, tout en ayant des distributions de capacité fondamentalement différentes, sont à même de lutter symboliquement et médiatiquement selon un simulacre d'égalité, change le principe de l'affrontement duel entre deux adversaires de même nature. Il faut penser simultanément la différence de type d'acteurs, leur multiplicité, leur autonomie, bref il faut admettre l'hétérogénéité des acteurs qui empêche de présupposer une identité au delà des différences de degré, identité finissant par réduire à deux joueurs les coalitions existantes.

8 Ainsi, les gouvernements luttent contre des organisations armées sans grand soutien populaire ( Sentier Lumineux...), contre des groupes mafieux (Cartel de Medellin et de Cali...), contre des organisations clandestines d'une dizaine d'hommes mais qui peuvent déstabiliser leur politique ( attentats de réseaux libanais ou algériens en France). Bien qu'infimes, ces acteurs agressent dans leurs fondements les États en remettant en cause leur prétention à être les seules sources de pouvoir légitime sur un territoire délimité par des frontières ${ }^{14}$. Cette attaque plus symbolique que militaire est souvent mal analysée. On a tendance à surestimer les capacités des organisations, à leur prêter des ressources cachées, à confondre l'impact symbolique avec la puissance effective. Or, ces acteurs sont pluraux et pas forcément interconnectés (à travers un ou des fils rouges ou 
verts...), ce qui fait que le gouvernement peut avoir de multiples micro-conflits simultanément plutôt qu'une mobilisation certes massive mais identifiable. Bien sûr, on trouvera toujours des auteurs pour vouloir ramener ces formes à une géopolitique classique où ils serviraient un adversaire caché mais puissant : hypothèse du fil rouge, des réseaux de la terreur, de la camorra mondiale, des nouvelles superpuissances du crime... mais il apparait à tous que cette utilisation de la matrice guerrière duelle est caricaturale dans l'explication de ces formes particulières de conflictualité dont un des aspects est justement le faible enracinement territorial qui les animent et la grande multiplicité des acteurs. La matrice guerrière appliquée à ces conflits sert avant tout à rassurer les gouvernants sur leur pérennité et à justifier des rhétoriques à l'égard de la population (nous ne négocions pas avec les terroristes...). On peut aussi chercher inversement à assigner ces formes de violence à des péripéties sans importance, réglables par la simple répression policière et par le " jugement " de l'État, ce que cherchent à faire les gouvernements européens, par exemple. Mais cette tentative de délégitimation de tout recours à la violence à l'intérieur de l'espace européen qui réussit pour les groupes clandestins internes se heurte au problème de la transnationalisation. Oscillant de la matrice de la guerre à celle du crime puni par la loi, personne ne sait encore comment analyser cette classe de phénomènes hétérogènes que par confort intellectuel on intitule terrorisme international ${ }^{15}$. L'hétérogénéité de ces phénomènes et leur flexibilité compliquent sérieusement l'analyse et rendent obsolètes les typologies générales fondées sur les analyses de contenu de l'idéologie, ou sur le contexte international, ou sur la géopolitique... Pour les comprendre, il faut pouvoir penser la transnationalité de certaines formes de conflits en s'arrachant au primat du paradigme réaliste et à celui de la géopolitique. Il faut analyser l'autonomie de micro acteurs collectifs par rapport au système étatique.

Le jeu des concurrences infra-étatiques et l'oubli de la conquête Les concurrences entre ces acteurs non gouvernementaux peuvent parfois être aussi déterminantes que leur relation au gouvernement pour comprendre leurs modalités d'action violente. Les alliances sont rares, le quotidien est celui des luttes pour la prééminence sur un objet référence parfois réduit concrètement à quelques sites (quartiers d'une ville, campus universitaire, parfois de simples squats). Il y a là une question d'échelle empêchant de parler d'une polarisation duelle. La lutte pour le pouvoir central peut être marginale, au regard d'objectifs beaucoup plus limités, même si leurs discours affirment le contraire. Il en va de même de leur relation à la population qu'il ne s'agit pas nécessairement d'encadrer, de contrôler ${ }^{16}$. Survivre comme groupe combattant, exister en soi sont des objectifs qui priment sur la conquête du pouvoir. Même lorsque la cause est perdue, voire oubliée, on continuera à se battre pour exister face aux autres microacteurs. Peu importe le gouvernement dans ce cas. C'est une microphysique des rapports de force qui s'instaure de facto et ruine la montée aux extrêmes, l'escalade, la réduction duelle. De plus, dans certains conflits, l'identification même d'un acteur comme acteur gouvernemental pose problème. Les logiques de fragmentation font qu'aucun acteur n'agit plus quasiment au niveau macro ou stratégique. Il n'y a plus que des multiplicités sans centre de référence. Même le fait de tenir la capitale ne suffit plus à polariser les combats comme semblent le montrer le Libéria et la Somalie. Comment alors reconnaître les acteurs, les identifier, les discriminer des non combattants? Comment penser les sujets stratégiques?

10 L'invisibilisation comme tactique, le sacrifice comme stratégie A certains moments, l'analyste y échoue malgré les tentatives de se faire valoir de tous les groupes. A 
d'autres, il est prisonnier de tactiques, où, les acteurs, loin d'être identifiés, demeurent invisibles ou clandestins ce qui ruine le principe de la stratégie militaire et tend à transformer la tâche du combat en tâche de renseignement, d'enquête policière. Le problème n'est plus l'affrontement et l'accumulation des forces mais l'identification du groupe qui a commis des actes de violence. Si nombre d'actes de violence commis par ces organisations revendiquent un territoire, on doute en permanence des enjeux du conflit et personne ne sait si l'on se bat bien pour la même chose. Les perceptions des acteurs non gouvernementaux sur les enjeux des conflits ne sont pas (et n'ont sans doute jamais été, même à la période bipolaire) analysables au sein du même univers social et stratégique que celui des acteurs dits centraux. Le territoire est parfois secondaire aux yeux des organisations et c'est avant tout une lutte pour la reconnaissance qui détermine l'enjeu du conflit ${ }^{17}$. Le conflit glisse de l'Avoir au Paraître, de l'Accumulation et de la Dette à l'Honneur et à la Honte ${ }^{18}$. Même la victoire militaire ne semble pas déterminante dans certains cas, où, paradoxalement la défaite militaire peut-être une ressource utile au sein d'une stratégie sacrificielle, plus intéressée par la mobilisation symbolique que par la conquête territoriale. Cette dimension sacrificielle de la mobilisation symbolique reste encore à explorer. Combien de groupes sacrifient délibérément leurs faibles effectifs pour faire parler d'eux? Quelle logique autre que l'économie des forces se révèle à cette occasion ? ${ }^{19}$

11 La délocalisation et le jeu des réseaux Les constitutions d'identités à cheval sur des frontières (kurdes mais aussi coptes ou basques) ou ayant des allégeances religieuses, ethniques, nationales transfrontières font que, dans ces cas, le territoire n'est plus en tant que tel un objet de conquête par la force mais un lieu d'expression d'une violence cherchant à prouver une existence. Dès lors, la délocalisation des actions de violence est tout à fait imaginable. On ne frappera pas sur le territoire revendiqué, ni même sur celui de l'adversaire mais " en terrain neutre ". Ces conflits se passent alors entre des réseaux d'acteurs en concurrence qui possèdent chacun des moyens de lutte et des ressources dépassant la plupart du temps le strict cadre " territorial-national-étatique " revendiqué. Ce sont des conflits où la lutte pour la reconnaissance ne passe plus forcément par le territoire. Les acteurs locaux non gouvernementaux ont profité de cet au delà du territoire revendiqué pour chercher à accroître leurs possibilités d'actions, multipliant ainsi les contacts à l'étranger, et la diplomatie active pour ne pas dépendre d'une seule source d'approvisionnement ${ }^{20}$. Même si la bipolarité a limité relativement leur possibilité et a impliqué un certain patronage, la relation patron client était loin d'être univoque et le patron a souvent été " obligé " d'agir pour satisfaire les acteurs locaux. Certains de ces réseaux d'acteurs ont innové en matière de répertoire d'action : $\mathrm{Au}$ local, patronné puis au " central-national " succéderait alors un troisième répertoire d'action à la fois transnational et patronné à l'échelle internationale ${ }^{21}$. Le national ne serait plus forcément la structure d'opportunité pertinente et plus important encore, la centralité perdrait de sa force d'attraction. Les groupes combattants diasporisés ou qui avaient perdu un ancrage territorial, ont vu dans ces pratiques de violence déconnectées d'objectifs militaires leur seule chance de modifier en leur faveur le rapport de force les opposant à un ou plusieurs adversaires gouvernementaux en jouant sur le symbolique et en impliquant des acteurs qui ne se sentaient nullement concernés. Ils ont inventé de nouvelles modalités d'actions violentes, de plus en plus déconnectées des enjeux de conquête territoriale qui réglaient les formes classiques de guerre. Modalités de plus en plus capillaires, moléculaires au lieu d'être des processus d'agrégation des forces et de mobilisation massive ${ }^{22}$. Cet " au delà du national ", au delà 
du territoire, n'est pas une nouveauté absolue mais sa fréquence n'a cessé d'augmenter depuis les années 60 . Seulement, malgré l'augmentation des travaux de terrain, nous devons reconnaître que nous ne savons toujours pas vraiment comment s'articulent les luttes territoriales et les luttes des réseaux, comment la microphysique des rapports de force s'expanse dans la mobilisation duelle ou se fragmente quasiment à l'infini. Comment passe-t-on des formes conflictuelles territorialisées à des formes conflictuelles en réseaux (et inversement)? Quelles en sont les modalités? La concurrence des réseaux peut renforcer à certains moments la stratégie de territorialisation (par exemple en Afghanistan) tout comme l'exacerbation des questions d'identités non territorialisées peut amener à la constitution de réseaux d'acteurs délocalisés mais actifs dans le conflit (diaspora kurde ou palestinienne...). Souvent la lutte pour la reconnaissance d'identités " fusionne " lutte territoriale militaire et lutte symbolique, activité de combat identifiable à un groupe et activités politiques d'une myriade d'acteurs difficilement repérables et usant de violence. La capillarisation de la violence, sa fragmentation est sans doute le trait le plus significatif de ce que certains sociologues ont appelé la post-modernité. Le défi lancé par certains acteurs individuels en dehors de toute possibilité de gagner dans un rapport de force avec les gouvernants ou leurs autres adversaires s'institue comme mode d'expression d'un rejet global d'un système de gouvernement et d'une forme de société. Les acteurs de ces conflits ( tantôt armées régulières mais souvent factions politiques, mouvement de foule, groupes actifs, organisations clandestines, milices, minorités combattantes) sont alors dépendants des structures de jeux politiques internes qui clivent les usages de la force, et de jeux politiques transnationaux qui se prolongent au delà du territoire à travers une géographie des sentiments, des solidarités et non de voisinage ${ }^{23}$.

Lire la suite

\section{NOTES}

1. Non appliquée aux analyses de la sécurité dite extérieure, cette hypothèse de la semiautonomie qui rompt avec les discours des acteurs qui vivent leur métier comme une stricte adaptation aux menaces a été développée dans le travail de Mary McIntosh, The organisation of crime, London, Macmillan, 1975.

2. Didier Bigo, " Grands débats pour un petit monde ", Cultures \& Conflits (" Troubler et inquiéter les discours du désordre international "), n 19/20, hiver 1995.

3. Sur le rapport entre les énoncés et la visibilité voir Michel Foucault, Naissance de la clinique PUF, 1966, et L'archéologie du savoir, Paris, NRF, Gallimard, 1969. Voir aussi l'ouvrage de Gilles Deleuze, Foucault, Paris, Minuit, 1986.

4. Nous serons volontairement très court sur ce point qui a été analysé en détail par Bertrand Badie et Marie Claude Smouts dans le chapitre sur la montée des flux transnationaux dans leur ouvrage Le retournement du monde : sociologie de la scène internationale, Paris, PFNSP, 1992. A quelques nuances près nous souscrivons à leur analyse qui synthétise une longue littérature. 
5. On en trouvera un résumé dans Gérard Chaliand, Anthologie mondiale de la stratégie, Paris, Robert Laffont, Coll. Bouquins, 1990. Le livre de Raymond Aron reste sur le plan théorique un des plus achevés : Raymond Aron, Penser la guerre, Clausewitz, Paris, NRF, Gallimard, 1976.

6. Gaston Bouthoul, Traité de Polémologie, Paris, Payot 1964

7. Voir l'ouvrage fondamental de Kalevi Holsti, Peace and war : armed conflicts and international order 1648-1989, Cambridge, Cambridge University Press, 1991. Sur l'impératif territorial voir ici même Denis Retaillé.

8. Cf. Charles Tilly, Coercion and capital from 990 to 1990, Blackwell, 1991. Tilly faisant rupture ici avec les approches en terme technologique sur les progrès des armes ou en terme strict de géopolitique.

9. B. Badie, La fin des territoires, Paris, Fayard, 1995 ; Michael Mann, " Nation-states in Europe \& other continents : diversifying, developing, not dying " in Deadalus, Summer 1993.

10. Outre les travaux classiques de Small Melvin et Singer, Resort to arms. International and civil wars 1816-1980, Sage, California, 1982. M. Brecher, Crisis and change in world Politics, 1986. Du SIPRI Yearbook, on citera les travaux récents et convergents de Peter Wallesrtein, Conflicts in the 1990, Uppsala University, 1994 ; d'Alex P Schmid et Bertho Jongman " Contemporary Armed Conflicts : a global inventory " in Pioom newsletter and report, summer 1994 and winter 1995, d'André Miroir in Les conflits armés de 1945 à nos jours CERIS, ULB 1995, et de Klaus Jurgen Gantzel (AKUF) 1995.

11. Outre les travaux classiques de Small Melvin et Singer, Resort to arms. International and civil wars 1816-1980, Sage, California, 1982. M. Brecher, Crisis and change in world Politics, 1986. Du SIPRI Yearbook, on citera les travaux récents et convergents de Peter Wallesrtein, Conflicts in the 1990, Uppsala University, 1994 ; d'Alex P Schmid et Bertho Jongman " Contemporary Armed Conflicts : a global inventory " in Pioom newsletter and report, summer 1994 and winter 1995, d'André Miroir in Les conflits armés de 1945 à nos jours CERIS, ULB 1995, et de Klaus Jurgen Gantzel (AKUF) 1995.

12. Outre les travaux classiques de Small Melvin et Singer, Resort to arms. International and civil wars 1816-1980, Sage, California, 1982. M. Brecher, Crisis and change in world Politics, 1986. Du SIPRI Yearbook, on citera les travaux récents et convergents de Peter Wallesrtein, Conflicts in the 1990, Uppsala University, 1994 ; d'Alex P Schmid et Bertho Jongman " Contemporary Armed Conflicts : a global inventory " in Pioom newsletter and report, summer 1994 and winter 1995, d'André Miroir in Les conflits armés de 1945 à nos jours CERIS, ULB 1995, et de Klaus Jurgen Gantzel (AKUF) 1995.

13. Voir la liste des instituts les publiant en note 10.

14. G. Poggi, The development of modern state, London, Hutchinson 1978, A. Giddens, The nation state and violence, Cambridge Polity Press, 1985. Sur le terrorisme :

Bertrand Badie, " Terrorisme et État " in Études Polémologiques, n 49, 1988.

15. Didier Bigo, Daniel Hermant, " Simulation et dissimulation. Les politiques de lutte contre le terrorisme en France ", Sociologie du Travail, octobre 1986.

16. Sans doute une approche mettant l'accent sur les jeux triangulaires entre d'une part l'affrontement duel des acteurs militarisés et d'autre part leur relation aux populations est-elle plus pertinente que la matrice guerrière. Julien Freund en avait donné les prolégomènes. Mais le modèle du triangle est lui-même trop restreint.

17. Sur ce point une illustration en est donné par Xavier Bougarel, " État et communautarisme en Bosnie-Herzégovine " in : Cultures \& Conflits, (" Communautarisme et Etat ") n 15/16, hiver 1994. La mise en récit de cette volonté de 
reconnaissance et la mobilisation des hommes pouvant parfois porter sur des actes passés (génocide arménien).

18. François Tricaud, L'accusation : recherche sur les figures de l'agression éthique, Paris, Dalloz, 1977.

19. Sur les stratégies sacrificielles : Didier Bigo et Daniel Hermant " La relation terroriste " in Études Polémologiques, n 47, 1987 ; Guy Nicolas : " Victimes ou martyrs " in : Cultures \& Conflits (" Interventions armées et causes humanitaires "), n 11, Automne 1993 ; Guy Nicolas Du don rituel au sacrifice suprême, Mauss/La Découverte 1996. Bertrand Badie, " La crise du golfe et le transnational " in Revue du monde musulman et méditerranéen, Aix-en-Provence, Mars 1991. Frederic Charillon " La connivence des acteurs non étatiques dans la guerre du Golfe " in Ariel Colonomos (dir.), " Sociologie des réseaux transnationaux " Paris, l'Harmattan, 1995. La guerre du Golfe a été à cet égard bien plus que l'invasion du Koweït et un conflit territorial. Côté Sadam Hussein, elle a mobilisé des espaces sociaux transnationaux et a joué dans les tous derniers moments d'une stratégie sacrificielle.

20. Dès les années 70 et surtout de 80 à 89 aucun groupe combattant conséquent ne dépend d'une seule source de financement. Ils diversifient leurs sources. Ce qui est vrai sur le plan financier l'est aussi sur le plan idéologique. Les grandes puissances ne contrôlent pas les acteurs locaux. Elles sont prisonnières elles-mêmes des tissus de relations que ceux-ci créent. Les interventions souvent non désirées de ces grandes puissances en disent d'ailleurs long sur leur faible maîtrise de la situation. Tous les travaux de sociologie des guerres le montre mais le courant " stratégiste " est si puissant à l'époque, en particulier dans sa variante globaliste, que ceci est ignoré ou plus exactement méconnu car cela remet en cause le principe même du raisonnement réduit à deux acteurs ; seul raisonnement permettant d'appliquer avec quelque crédibilité la formalisation mathématique et la théorie des jeux en vogue à l'époque. 21. Ceci serait une transformation forte des répertoires d'action contestataire euxmêmes que Charles Tilly avait synthétisé dans La France conteste de 1600 à nos jours, Paris, Fayard 1986.

22. Le fait que les enjeux de conquête territoriale ne soient plus primordiaux et que le système international de reconnaissance juridique des frontières des États les uns par les autres aient " clôt " les possibilités de conquête (Cf. invasion du Koweït) n'a pas encore été assez analysé. Il entraîne une rupture dans les formes de la guerre et dans ses buts.

23. Une table ronde de Cultures \& Conflits fut consacrée à ce thème. Sur la Yougoslavie voir le texte stimulant de Xavier Bougarel " État et communautarisme en BosnieHerzégovine ", Cultures \& Conflits, " Communautarisme et État ", nº 15/16 Hiver 1994, ainsi que son article dans Confluences méditerranée, Printemps 1995.

\section{INDEX}

Mots-clés : conflits, guerre, Relations Internationales, réseaux transnationaux, sociologie des conflits (polémologie), territoire(s) et territorialité 